\title{
Curricula for Teaching MRI Safety and MRI/CT Contrast Safety To Residents: How Effective Are Live Lectures and Online Modules?
}

\author{
Corresponding author: \\ Jordan Swensson, MD \\ Indiana University School of Medicine Department of Radiology and Imaging Sciences, \\ University Hospital Rm 0641 \\ 550 N. University Blvd. \\ Indianapolis, IN 46202 \\ jswensso@iupui.edu \\ phone 317-948-2449 \\ fax 317-948-2803 \\ Co-Authors (all from Indiana University School of Medicine, Indpls., IN): \\ Lane McMahan, MD \\ Ben Rase, MD \\ Bilal Tahir, MD
}

All the authors receive no support in the form of grants, equipment, drugs, or other assistance. The authors have no conflicts of interest to disclose.

\section{KEY WORDS}

Education, MRI, CT, Contrast, Residents

\section{SUMMARY SENTENCE}

Our study demonstrates that live lectures and online modules are equally effective in teaching residents about CT and MRI contrast and safety. This allows residency programs flexibility in the educational intervention that works for their needs.

This is the author's manuscript of the article published in final edited form as:

Swensson, J., McMahan, L., Rase, B., \& Tahir, B. (2015). Curricula for Teaching MRI Safety, and MRI and CT Contrast Safety to Residents: How Effective Are Live Lectures and Online Modules? Journal of the American College of Radiology, 12(10), 1093-1096. http://dx.doi.org/10.1016/j.jacr.2015.04.012 


\section{ABSTRACT}

Purpose

The advent of the diagnostic radiology Core Exam and the new ACGME Milestone evaluation system for radiology residents places new emphasis on topics in MRI and CT safety and MRI and CT contrast agents. We evaluated whether lecture-based teaching or online modules would improve baseline resident knowledge in these areas, and assessed which intervention was more effective.

\section{Methods}

Prior to didactic intervention, two cohorts were created from 57 radiology residents with equal numbers and matched level of training. The residents were tested on their baseline knowledge of MRI, MRI contrast safety, and CT contrast safety with a multiple-choice examination. One group attended a live, one hour lecture on the above topics. The other engaged in three short, online educational modules. After six weeks, the residents were again tested with the same questions to assess for improvement in their understanding.

\section{Results}

Both the module and lecture cohorts demonstrated a statistically significant increase in questions answered correctly on CT contrast safety (13.1\%, $\mathrm{p}<0.001$, and $19.1 \%, \mathrm{p}<0.001$ respectively) and on MRI/MRI contrast safety (12.9\%, $\mathrm{p}<0.001$, and $14.4 \%, \mathrm{p}<0.001)$. The pre-intervention and post-intervention scores, and degree of improvement post-intervention was similar for the module vs lecture groups without statistical difference $(p=0.70)$. Resident confidence improved in both groups for both modalities. 


\section{Conclusions}

Focused didactic intervention improves resident knowledge on issues of MRI and CT safety and MRI and CT contrast agents. Live lectures and online modules can be equally effective tools, allowing residency programs flexibility. 


\section{INTRODUCTION}

The demands on today’s radiology residency programs are continuing to evolve and expand. Each program faces the challenges and opportunities inherent in resident education in the nascent stages of the American Board of Radiology (ABR) Core Examination ${ }^{1}$. To this end, residency programs affiliated with the American Council for Graduate Medical Education (ACGME) have been provided with a new framework for evaluating residents: a joint project with the ABR known as the Diagnostic Radiology Milestone Project ${ }^{2}$. The goal of the Milestone Project is to provide a framework to help program directors assess residents’ progress in multiple areas of clinical and professional competency.

The new evaluation framework will require residency programs to re-evaluate the way in which resources are proportioned to facilitate the goals of the milestone project. One area that will likely present a challenge is the teaching and evaluation of non-interpretive skills, an area of professional development that has traditionally been assigned a lower priority. To address this new evaluation paradigm, residency programs have developed and will continue to develop new models and tools to aid their work. Our interest lies in the sub-area of the Milestones of Practice-based Learning and Improvement, specifically patient safety as it relates to contrast agents, radiation safety, MR safety, and sedation.

A variety of work has been done in the past to explore the idea of educating residents in the areas of MRI physics and to a lesser degree MRI safety, as well as in regard to CT contrast and safety ${ }^{3,4,5}$.

To our knowledge, no targeted research with the Milestones in mind has been done to evaluate effective ways to educate residents in these topics. Here we present data on our efforts 
to objectively measure the utility of online modules and traditional didactic lectures in teaching these important milestone concepts. More specifically we measured overall improvement in knowledge and subjective comfort when using online modules and didactic lectures to teach the same material.

\section{METHODS AND MATERIALS}

Our study was carried out at our single, large, academic radiology residency program. A total of 57 radiology residents spanning all levels of resident radiology training (year one through year four) participated. At the outset, the residents were split into two cohorts that were matched for year of training [Table 1]. A set of 42 questions was designed by the study authors to cover the areas of MRI safety, MRI contrast and contrast use safety, and CT contrast and contrast use safety. Specific topics included types and doses of MRI and CT contrast, contrast reactions, contraindications, and MRI department zones [Figure 1].

These questions were then presented via online survey tools to the residents. These data were used to establish a knowledge baseline among the two cohorts. An additional question regarding the residents' subjective comfort in their knowledge about the areas above was

included, using a five point Likert scale with five representing the highest degree of comfort. Prior to the education intervention, a set of three modules were created by the study authors, each focusing on one of the areas described above. The material included in the modules was taken primarily from the ACR Guidance Document for MR Safe Practices ${ }^{6}$, the ACR Manual on Contrast Media ${ }^{7}$, and from the ABR's study guide for the residency core examination. 
These modules were created using Microsoft PowerPoint [TM] and then transferred to Adobe Captivate [TM]. One of the study authors then hosted the modules on a website.

Following this, one cohort was given access to the online modules and instructed to complete them at their own pace over the course of approximately one week. The other cohort was instructed to attend a one hour live lecture during dedicated didactic time during the work day. This lecture was provided by the study authors and contained identical information to that compiled in the online modules.

Approximately six weeks following these educational interventions, the same set of questions was administered to the lecture and module cohorts. The residents' subjective comfort level with the material was reassessed. The pre and post intervention data was collected and evaluated. A paired t test was used to evaluate for statistically significant improvement of the groups with intervention. An unpaired equal variance test was performed to evaluate performance and improvement between the cohorts.

\section{RESULTS}

50 of the 57 residents completed the entire CT study, and 51 of the 57 residents completed the entire MRI study. Those who did not complete the entire study did not take one of the pre or post intervention tests, and their data was excluded. Following data collection and statistical analysis as above, the results of questions pertaining to CT contrast/safety and MRI contrast/safety were separated, and are reproduced in [Table 2] [Table 3].

There was no statistically significant difference in cohort performance prior to the lecture or modules. The performance of the residents in the lecture and module cohorts after the educational intervention was then compared. 
In the area of CT contrast/safety, the lecture cohort demonstrated an absolute improvement of 19.2 percent in percentage of questions answered correctly following the educational intervention $(\mathrm{P}<0.001)$. The module cohort demonstrated an absolute improvement of 23.1 percent in percentage of questions answered correctly $(\mathrm{P}<0.001)$. There was no statistically significant difference in the percentage correct between the module or lecture cohorts in either the pre or post intervention raw scores.

In the area of MRI contrast/safety, the lecture cohort demonstrated an absolute improvement of 14.5 percent in percentage of questions answered correctly following the educational intervention $(\mathrm{P}<0.001)$. The module cohort demonstrated an absolute improvement of 12.9 percent in percentage of questions answered correctly $(\mathrm{P}<0.001)$. There was no statistically significant difference in the percentage correct between the module or lecture cohorts in either the pre or post intervention raw scores.

There was no statistically significant difference between the degree of improvement in the lecture group compared to the module group in either CT contrast/safety or MRI contrast/ safety, $\mathrm{p}=0.82$ and $\mathrm{p}=0.43$ respectively.

The residents expressed an statistically significant increase in their relative comfort level with issues of CT and MRI contrast/safety on a five point Likert scale, from 2.97 to 3.55 on CT $(\mathrm{P}<0.005)$ and 2.65 to 3.28 on MRI $(\mathrm{P}<0.005)$.

\section{DISCUSSION}

Our work was designed to evaluate the effectiveness of live lectures and online modules in teaching residents topics related to CT and MRI contrast and safety. The above results demonstrate that both the designed lectures and modules led to a statistically significant 
improvement in resident knowledge of these areas. This improvement was durable, as the residents were not asked to recall what they had learned until six weeks following the educational interventions. The residents also felt that the interventions improved their confidence regarding these topics. And, perhaps most interestingly, no statistically significant difference was found in the degree of increase in knowledge between the lecture and the module cohorts.

These results demonstrate that either live lectures or online modules can be effectively used to teach important concepts in radiology education. The equality between the educational methods suggests that programs can utilize either or both of the methods to their advantage without sacrificing efficacy. The unique demands on each program's residents, staff physicians, and support staff mean that resident education will by necessity differ among programs. As an example, the feasibility of dedicated lecture time during the day may be difficult or impossible for some programs depending on their size, culture and workflow. An online, "at your own pace,” educational option may work better for these programs, or even for programs with dedicated lecture time that don't have room in their schedules. Conversely, those programs with lecture time built in may feel more comfortable sticking with their didactic methods, making live lectures a desirable option.

A possible added benefit to the online modules is in the relative ease of accessing and sharing this information. Dedicated educational material such as what was developed for this study can be readily published either by conventional methods for profit or by more open means through sundry video sharing websites. The availability of such tools could led to a more uniform resident educational experience across multiple institutions, and perhaps motivate continuing improvement in the educational materials themselves. 
Both live lectures and modules can also be useful to residency programs as part of their evaluation process of residents in the ACGME/ABR Milestones Project. At our institution, resident participation in the lecture or modules and the pre and post intervention questions was taken as a marker of achievement in the previously described Milestones sub-area of patient safety. Depending on program preference, individual resident performance on pre and post intervention questionnaires could also be used to guide targeted assistance to those residents who demonstrate difficulty with CT or MRI contrast or safety issues.

This study as presented does carry with it certain limitations. As the data collected came exclusively from multiple choice questions, only resident knowledge of, not practical execution of, the material presented can be assessed. Knowing by rote how to manage CT contrast reactions is a different manner than applying that knowledge to a patient on your table. This study focuses only on a single academic radiology residency. Also, it can only be said that the specific interventions designed for this study are effective at improving resident knowledge of CT and MRI contrast and safety. Generalization to material presented in other lectures or online modules cannot be assessed.

Nevertheless, the results of the study demonstrate that it is feasible to create and implement effective live or online educational tools to teach this vital information. In the future, we hope to continue to assess the longer term effectiveness of the interventions by re-evaluating the knowledge of our institution's residents even farther out from our intervention. The modules and lectures created have been and will continue to be used as part of our program's recurring resident education cycle, as well as to fulfill resident evaluation and promotion milestones. Finally, it is our aim to provide access to the educational tools that we have created for use by other residents and residency programs to assist in their continuing professional development, 
perhaps through our department's educational webpage or through an online streaming video site.

In conclusion, our study demonstrates that both live lectures and online modules are effective in teaching radiology residents about issues pertaining to CT and MRI contrast and safety. No statistically significant difference in the effectiveness of the lectures versus the modules was found, which means residency programs have flexibility in choosing they type of educational intervention that works best for their needs. Teaching CT and MRI contrast and safety is of vital importance for producing the next generation of able radiologists, as well as for preparing residents for the new ABR Core Exam and helping residency programs fulfill the requirements of the ACGME/ABR Milestones Project for resident evaluation.

\section{TAKE HOME POINTS}

- The ABR Radiology Core Examination and ACGME/ABR Milestone Project place new emphasis on non-interpretive skills such as MRI and CT safety

- Live lectures and online modules are equally effective didactic tools for increasing resident knowledge of MRI, MRI contrast safety, and CT contrast safety

- This will allow flexibility for residency programs as they continue to adapt and adjust their curricula for this new environment 


\section{REFERENCES}

1. The American Board of Radiology. American Board of Radiology Core Exam. http://www.theabr.org/ic-dr-core-exam. (Accessed 02/09/2015)

2. Accredidation Council for Graduate Medical Education / The American Board of Radiology. Diagnostic Radiology Milestone Project.

http://acgme.org/acgmeweb/Portals/0/PDFs/Milestones/DiagnosticRadiologyMilestones. pdf. (Accessed 02/09/2015)

3. Deitte, Lori. The New Residency Curriculum: Professionalism, Patient Safety, and More. J Am Coll Radiol. 2013;10:613-17

4. Zhuo, J., Gullapalli, P. MR Artifacts, Safety, and Quality Control. RadioGraphics. 2006;26:275-97

5. Nickoloff, E., Mahesh, M., Heintz P., et. al. Physics Instruction for Radiology Residents in the Era of the New ABR Examination Process. J Am Coll Radiol. 2010;7:900-4

6. Kanal, E. Barkovich J., Bell C., et. al., ACR Guidance Document for MR Safe Practices, J. Magn. Reson. Imaging. 2013;37:501-30.

7. American College of Radiology. Manual on Contrast Media Version 9, ACR Committee on Drugs and Contrast Media, 2013, http://www.acr.org/quality\%20safety/resources/ /media/37D84428BF1D4E1B9A3A2918DA9E27A3.pdf/. (Accessed 02/09/2015) 
Table 1.

\section{Demographics of Study Cohorts}

Lecture Cohort (\# of residents) Module Cohort (\# of residents)

PGY-2

PGY-3

PGY-4

PGY-5

Total
8

6

7

8

29
7

6

7

8

28 


\section{Figure 1.}

\section{Example Questions}

What is the primary danger associated with crossed limbs and looped medical leads/wires on a patient located in the bore of an MRI scanner?

a. Induced electric currents can lead to local overheating and burns

b. Increased artifact from these objects can lead to c. image degradation

d. Induced magnetic fields can lead to local image artifacts

e. There is no primary concern with either of these issues

In a patient with a history of adverse reaction to GBCA, which of the following best describes the risk for a second reaction following subsequent GBCA administration.
a. $2 x$
b. $5 x$
c. $8 x$
d. $15 x$

Contrast agents are frequently classified as having high or low osmolality relative to blood. Which of the follow agents has osmolality closest to that of blood?

a. Contrast agent A: $500 \mathrm{mOs} / \mathrm{kg}$

b. Contrast agent B: $300 \mathrm{mOsm} / \mathrm{kg}$

c. Contrast agent C: $100 \mathrm{mOsm} / \mathrm{kg}$

d. Contrast agent D: $50 \mathrm{mOsm} / \mathrm{kg}$ 
Table 2.

Data from CT contrast and safety questions

Cohort $\%$ Correct Before Intervention

Lecture $(n=\quad 53.0 \pm 12.6$

22)

Module $(\mathrm{n}=\quad 60.0 \pm 13.5$

28)

\begin{tabular}{l|l|l|}
$\begin{array}{l}\text { \% Correct After } \\
\text { Intervention }\end{array}$ & \% Change & $P$ Value \\
\hline $72.2 \pm 15.9$ & $19.2 \pm$ & $<.001$ \\
\hline $73.1 \pm 12.1$ & 16.2 & \\
\hline & $23.1 \pm$ & $<.001$ \\
\hline
\end{tabular}

\section{Table 3}

Data from MRI Contrast / Safety Questions

$\begin{array}{lll}\text { Percent Correct } & \text { Percent Correct } & \text { Percent Change }\end{array}$

\begin{tabular}{|c|c|c|c|c|}
\hline $\begin{array}{l}\text { Lecture Cohort } \\
(\mathrm{n}=20)\end{array}$ & $54.0+/-10.6$ & $68.5+/-11.3$ & $14.5+/-14.6$ & $<0.001$ \\
\hline Module Cohort & $53.0+/-9.7$ & $65.9+/-11.4$ & $12.9+/-13.0$ & $<0.001$ \\
\hline
\end{tabular}

